\title{
Trombose Tardia (3 Anos e 9 Meses) de Stent Cypher
}

\author{
Marco Antônio M. Fossati ${ }^{1}$, Arthur Valls B. Cavalcanti ${ }^{1}$
}

\section{RESUMO}

A trombose tardia nos stents revestidos com drogas antiproliferativas é uma realidade a ser enfrentada. Ela ocorre com maior freqüência, dependendo do critério de trombose, do que em stents convencionais. A trombose pode se dar anos após o implante e mesmo em pacientes usando antiagregante plaquetário. O desfecho clínico pode ser dramático, entretanto, não sabemos ainda se este evento realmente aumenta a mortalidade, quando comparado ao stent convencional. Os autores relatam um caso de trombose de stent Cypher, quase quatro anos após o seu implante.

DESCRITORES: Contenedores, efeitos adversos. Reestenose coronária. Trombose coronária, etiologia. Paclitaxel. Sirolimo.

0 implante de stents com eluição de drogas antiproliferativas (DES) faz parte da prática diária da intervenção coronariana. Estima-se que $80 \%$ dos pacientes submetidos a angioplastia coronária recebam DES, nos Estados Unidos e na Europa. A euforia com os primeiros resultados de acompanhamento tardio nos estudos RAVEL ${ }^{1}$ e TAXUS ${ }^{2}$, que mostravam perda tardia próxima a zero, deu lugar, hoje, à preocupação com eventos como a trombose tardia intra-stent ${ }^{3,4}$. Os autores relatam um caso de comprovada trombose intra-stent, em um paciente tratado com prótese com eluição de sirolimus, três anos e nove meses antes, não tendo encontrado na literatura relato de caso com esta evolução.

\section{RELATO DO CASO}

Paciente 60 anos, sexo masculino, portador de hipertensão arterial sistêmica e dislipidemia controladas com medicação. Em outubro de 2002, foi submetido a angioplastia e implante de stent Cypher 3,0 x $18 \mathrm{~mm}$

\footnotetext{
1 Serviço de Cardiologia do Hospital Moinhos de Vento de Porto Alegre, RS.

Correspondência: Marco Antônio M. Fossati. Rua Mariz e Barros, 392, Apto. 701 - Petrópolis - Porto Alegre - RS - CEP 90690-390.

E-mail: marcofossati@brturbo.com.br

Recebido em: 30/10/2006 • Aceito em: 12/2/2007
}

\section{SUMMARY}

Late Stent Thrombosis (3 years and 9 months) after Cypher Implantation

Late stent thrombosis in drug-eluting stents is a reality. It occurs more frequently with drug-eluting stents, depending on the criteria used, than with bare metal stents. Late stent thrombosis can take place years after implant, even in patients on platelet antiaggregant agents and the clinical result can be dramatic. However, it is still unclear if this event really increases mortality compared to conventional stenting. The authors describe a case of late thrombosis almost four years after Cypher implantation.

DESCRIPTORS: Stents, adverse effects. Coronary restenosis. Coronary thrombosis, etiology. Paclitaxel. Sirolimus.

(Figuras 1 e 2) a 18 atm, para tratar lesão de novo, excêntrica, de segmento proximal da artéria coronária direita. Estimou-se em 3,0 mm o diâmetro do vaso-alvo por análise $\mathrm{QCA}^{1}$. Procedimento realizado sem intercorrências, tendo o paciente recebido alta com suas medicações habituais acrescidas de AAS 100 mg/dia e clopidogrel $75 \mathrm{mg} /$ dia. Manteve-se com esquema duplo antiagregante plaquetário por nove meses, permanecendo com AAS 100 mg/dia após este período.

Em julho de 2006, compareceu à emergência de nosso hospital, com forte dor precordial, de início súbito, quatro horas antes. Após diagnóstico de infarto agudo do miocárdio de parede inferior, foi levado a cateterismo de urgência. Exame mostrou a artéria coronária esquerda sem lesões significativas e oclusão da artéria coronária direita, imediatamente antes do stent implantado anteriormente (Figura 3). Procedeu-se à recanalização mecânica com corda-guia 0,014 sem dificuldade e angioplastia com balão 3,5 x $20 \mathrm{~mm}$ a 9 atm. Angiografia após a primeira angioplastia mostrou embolização distal de grande carga de trombos (Figura 4). Diante deste quadro, foi realizada extração dos trombos com cateter Pronto. Não foram utilizados inibidores da glicoproteína Ilb/IIla. Angiografia final da artéria coronária direita (Figura 5) mostra coronária com fluxo TIMI 3 e ausência de estenose coronariana. O paciente recebe alta em uso de AAS 100 mg, Clopidogrel $75 \mathrm{mg}$ e sua medicação habitual. 


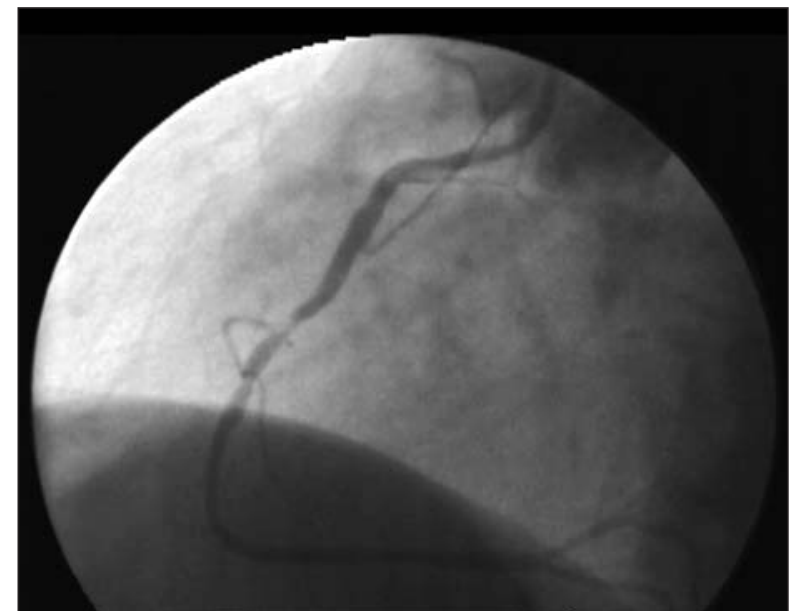

Figura 1 - Estenose importante de artéria coronária direita, em outubro de 2002 .

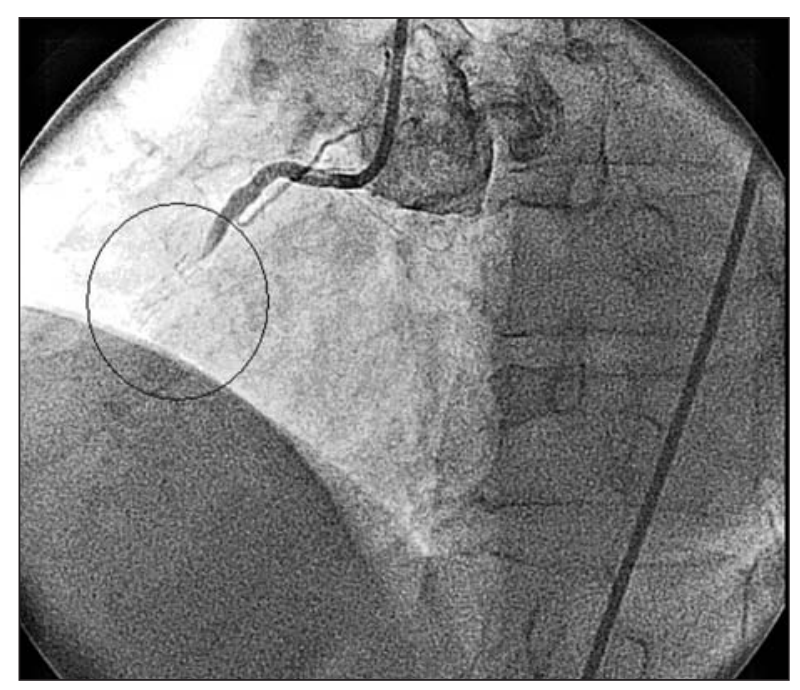

Figura 3 - Oclusão total tardia, em julho de 2006.

\section{DISCUSSÃO}

Trombose tardia intra-stent ocorre com maior freqüência em DES do que nos convencionais ${ }^{5}$. A razão estimada de trombose é de $0,2 \%$ a $0,4 \%$ ao ano, nos primeiros quatro anos. As causas da trombose tardia nos DES são multifatoriais, sendo que o atraso na endotelização arterial tem papel importante ${ }^{6}$. Nove meses após implante de um stent convencional, aproximadamente $100 \%$ encontram-se endotelizados, enquanto que não mais que $50 \%$ dos DES assim se encontram ${ }^{6}$. Além disto, o dimensionamento incorreto do tamanho da prótese e a inadequada aposição das hastes são fatores de risco para trombose. Do ponto de vista técnico, a recomendação é para a utilização da ultrasonografia intravascular intracoronária, principalmente pós-procedimento, e a expansão correta com insuflação, conforme a complacência do balão.

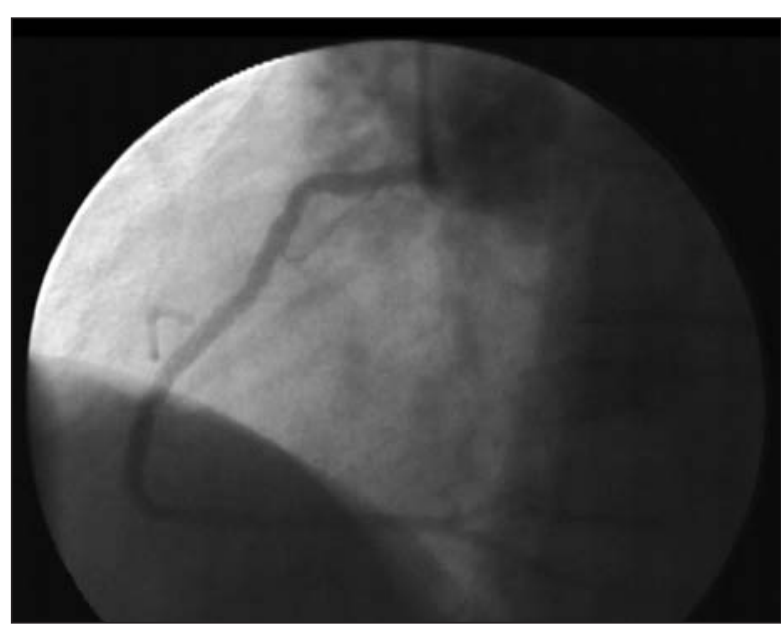

Figura 2 - Resultado final pós-implante de stent Cypher.

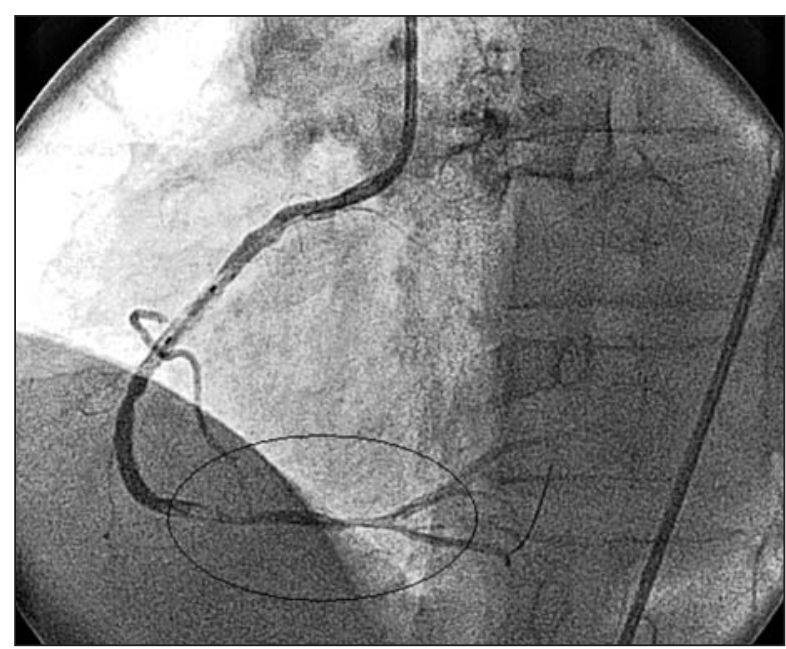

Figura 4 - Carga de trombos com embolização distal.

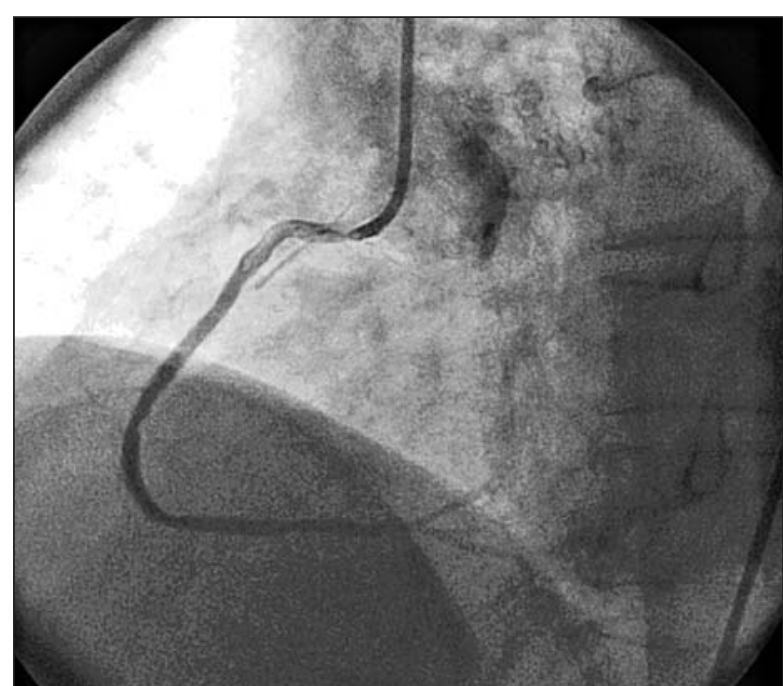

Figura 5 - Resultado final após ACTC, em julho de 2006. 
No caso relatado, cabe ressaltar a utilização do cateter Pronto para extração distal de trombos. Apesar de resultados conflitantes dos estudos já realizados, em relação ao benefício na recuperação do fluxo TIMI III no infarto agudo com o uso de tal instrumento (estudo DEAR-MI), ele é de fácil e segura utilização. Os autores, frente ao quadro dramático, utilizaram o dispositivo, sem complicações.

Algumas questões devem ser respondidas em relação à trombose tardia de DES:

1. quanto tempo é necessário de uso da terapia dupla antiplaquetária?;

2. existe realmente um aumento na mortalidade em pacientes que receberam DES?;

3. qual a definição de trombose intra-stent?;

4. o aumento do risco de morte relacionado à revascularização da lesão-alvo nos stents convencionais contrabalança o aumento de trombose nos DES?

\section{REFERÊNCIAS BIBLIOGRÁFICAS}

1. Morice MC, Serruys PW, Souza JE, Fajadet J, Ban Hayashi $\mathrm{E}$, Perin $\mathrm{M}$, et al. A randomized comparison of a sirolimus- eluting stent with a standard stent for coronary revascularization. N Engl J Med. 2002;346(23):1773-80.

2. Colombo A, Drzewiecki J, Banning A, Grube E, Hauptmann $\mathrm{K}$, Silber $\mathrm{S}$, et al. Randomized study to assess the effectiveness of slow and moderate release polymer-based paclitaxeleluting stents for coronary artery disease. Circulation. 2003; 108(7):788-94

3. Ong AT, Hoye A, Aoki J, van Mieghem CA, Rodriguez Granillo GA, Sonnenschein K, et al. Thirty-day incidence and six-month clinical outcome of thrombotic stent occlusion after bare metal stent, sirolimus and paclitaxel stent implantation. J Am Coll Cardiol. 2005;45(6):947-53.

4. McFadden EP, Stabile E, Regar E, Cheneau E, Ong AT, Kinnaird $\mathrm{T}$, et al. Late thrombosis in drug-eluting coronary stent after discontinuation of antiplatelet therapy. Lancet. 2004;364(9444):1519-21.

5. Pfisterer ME, Kaiser CA, Bader F, Brunner-La Rocca HP, Bonetti PO, Buser PT. Late clinical events related to late stent thrombosis after stopping clopidogrel: prospective randomized comparison between drug - eluting versus baremetal stenting. In: $55^{\text {th }}$ Annual Scientific Sessions of the American College of Cardiology. Abstracts. March 11-14, 2006, Atlanta, Georgia.

6. Joner M, Finn AV, Farb A, Mont EK, Kolodgie FD, Ladich $\mathrm{E}$, et al. Pathology of drug-eluting stents in humans: delayed healing and late thrombotic risk. J Am Coll Cardiol. 2006; 48(1):193-202. 